\title{
Bounds on new physics from electric dipole moments
}

\section{Martin JUNG*}

TUM Institute for Advanced Study / Excellence Cluster Universe,

Technische Universität München

E-mail: martin.jung@tum.de

Preprint: FLAVOUR (267104)-ERC-109

\begin{abstract}
Electric dipole moments are extremely sensitive probes for additional sources of $\mathrm{CP}$ violation in new physics models. The multi-scale problem of relating the high-precision measurements with neutrons, atoms and molecules to fundamental parameters can be approached modelindependently to a large extent; however, care must be taken to include the uncertainties from especially nuclear and QCD calculations properly. The resulting bounds on fundamental parameters are illustrated in the context of Two-Higgs-Doublet models.
\end{abstract}

Flavor Physics \& CP Violation 2015

May 25-29, 2015

Nagoya, Japan

${ }^{*}$ Speaker. 


\section{Introduction}

Electric dipole moments (EDMs) provide a competitive means to search for new physics (NP), complementary to strategies like direct searches at hadron colliders, but also to other indirect searches like the flavour-changing processes investigated at the flavour factories. The exceptional sensitivity is due to the combination of experimental precision with a tiny Standard Model (SM) background. The smallness of the latter is related to the very specific connection between flavour and $\mathrm{CP}$ violation in the SM, ${ }^{1}$ embodied by the Kobayashi-Maskawa mechanism [1], which is very effective in suppressing flavour-changing neutral currents (FCNCs) [2], and even more so in suppressing flavour-conserving ones involving $\mathrm{CP}$ violation. An exception is provided by the gluonic operator $\mathscr{O}_{G \tilde{G}} \propto \varepsilon_{\mu v \rho \sigma} G^{\mu v} G^{\rho \sigma}$, yielding a potentially very large contribution to hadronic EDMs which is, however, strongly bounded experimentally, constituting the strong CP problem. To explain its absence, typically symmetries are invoked, involving additional particles. The most famous example is the Peccei-Quinn mechanism [3], predicting the presence of axions [4, 5]. While these have not yet been observed, it is implicitly assumed in this work when discussing hadronic EDMs that the strong CP problem is solved by this or a similar mechanism. The remaining SM contributions then lead to EDMs many orders of magnitude below the present limits, e.g. $d_{n}^{\mathrm{SM}, \mathrm{CKM}} \lesssim 10^{-(31-32)} e \mathrm{~cm}[6-9]$. Importantly, for leptonic EDMs no assumption regarding $\mathscr{O}_{G \tilde{G}}$ is necessary; the SM contribution to the electron EDM is estimated to be $d_{e}^{\mathrm{SM}} \lesssim 10^{-38} e \mathrm{~cm}[10-12]$. The observation of an EDM with the present experimental precision would therefore clearly constitute a NP signal, especially in the leptonic sector.

Sakharov's conditions [13] require the presence of new sources of CP violation to explain the observed baryon asymmetry of the universe; while this does not imply sizable EDMs, they are generally very sensitive to such sources. In fact, generic NP scenarios usually yield contributions that are large compared to experimental limits, implying either a high NP scale or a very specific structure for additional CP-violating contributions, similar to the situation in the flavour-changing sector. Casting these qualitative statements into reliable bounds on model parameters requires knowledge of their relation to the experimental observables - typically (bounds on) frequency shifts obtained for composite systems. The calculation of these relations proceeds via a series of effective field theories (EFTs), see e.g. Refs. $[14,15]$ for recent reviews and references therein. Importantly, this approach allows to perform a large part of the analysis model-independently. The calculation of the matrix elements of the corresponding effective operators often include large uncertainties which have to be taken into account, see Refs. $[15,16]$ for recent detailed discussions. Furthermore, in composite systems different contributions can exhibit cancellations; this issue can already be systematically addressed for paramagnetic systems [17,18], and in the future potentially also for diamagnetic ones [19].

This article proceeds as follows: model-independent constraints are discussed in the following section, focusing on paramagnetic systems. In Sec. 3 NP contributions to EDMs are discussed first generally, then using Two-Higgs-Doublet models (2HDMs) as a specific example. We conclude in Sec. 4 .

\footnotetext{
${ }^{1}$ EDMs are T,P-odd; their existence implies also CP violation when assuming CPT to be conserved as we will in this article.
} 


\section{Model-independent constraints from EDM measurements}

The available competitive observables, that is, the EDMs of thorium monoxide (ThO) and ytterbium fluoride (YbF) molecules [20,21], thallium $(\mathrm{Tl})$ and mercury $(\mathrm{Hg})$ atoms [22,23] and the neutron [24,25] (see also [26]), are related by calculations on the molecular, atomic, nuclear and QCD levels to the coefficients of an EFT at a hadronic scale (see, e.g., Refs. [14, 15, 27]):

$$
\mathscr{L}_{\mathrm{eff}}^{\mathrm{EDM}}=-\sum_{f} \frac{d_{f}^{\gamma}}{2} \mathscr{O}_{f}^{\gamma}-\sum_{q} \frac{d_{q}^{C}}{2} \mathscr{O}_{q}^{C}+C_{W} \mathscr{O}_{W}+\sum_{f, f^{\prime}} C_{f f^{\prime}} \mathscr{O}_{f f^{\prime}} .
$$

This operator basis consists of (colour-)EDM operators $\mathscr{O}_{f}^{\gamma, C}$, the purely gluonic Weinberg operator $\mathscr{O}_{W}$ and T- and P-violating four-fermion operators $\mathscr{O}_{f f^{\prime}}$ without derivatives $\left(f^{(\prime)}=e, q, q=u, d, s\right)$. Since these calculations do not depend on the NP model under consideration, this Lagrangian is used as the interface between experiment and high-energy calculations: the latter provide the model-specific expressions for the Wilson coefficients in Eq. (2.1), with at least one more intermediate EFT at the electroweak scale.

In neutral composite systems, the EDMs of the components are shielded; for non-relativistic, point-like constituents this shielding is perfect, therefore measurements for this type of system rely on the violation of these assumptions [28]. For paramagnetic systems, relativistic effects can actually lead to enhancement factors, if the proton number $Z$ is large enough [29-31], since two contributions scale approximately with $Z^{3}$ : these are the ones from the electron EDM and the scalar electron-nucleon coupling, $\tilde{C}_{S} .{ }^{2}$ Heavy paramagnetic systems can therefore be assumed to be completely dominated by these two contributions, allowing a model-independent fit to bound and eventually determine both contributions, without the assumption of a vanishing electron-nucleon contribution $[17,18]$. In practice, there are two complications with this approach at present, which can however be overcome with additional measurements. Firstly, the ratio of the coefficients of the two contributions is necessarily similar for heavy paramagnetic systems [17]. This problem can be solved by performing measurements with atoms or molecules with largely different proton numbers, such as rubidium and francium atoms. Absent such (competitive) measurements, it is possible to assume e.g. the limit from $\mathrm{Hg}$ to be saturated by the $\tilde{C}_{S}$ contribution [18]: this is a conservative procedure, since the EDM of this system is typically dominated by colour-EDM (cEDM) contributions. While this lead in the past to a similar limit on $d_{e}$ like naively setting $\tilde{C}_{S} \rightarrow 0$, the unmatched precision of the recent ThO measurement (in the $d_{e}-\tilde{C}_{S}$ plane) poses the second problem, namely that the projections of the 2-dimensional fit remain almost unchanged, see Fig. 1 (left). Since this may appear overly conservative, a temporary option is to limit the cancellation between the two contributions within the ThO measurement, i.e. to allow the $\tilde{C}_{S}$ contribution to at most saturate the experimental limit $n=1,2,3, \ldots$ times, see Fig. 1 (right), as a compromise between the conservative procedure described previously and the potentially critical assumption of $\tilde{C}_{S}=0$. The corresponding fits lead to

$$
d_{e} \leq 1.0(0.16) 10^{-27} e \mathrm{~cm}(95 \% \mathrm{CL})
$$

\footnotetext{
${ }^{2}$ Note that $\tilde{C}_{S}$ depends in general on the considered system. However, for the systems at hand (and more generally for heavy paramagnetic systems), it is universal to very good approximation [18].
} 
using $\mathrm{Hg}$ and $n=1$, respectively. These values should be used when extracting bounds on parameters from $d_{e}$ in any model in which the electron-nucleon contribution cannot be argued to be negligible. Furthermore, these 2-dimensional constraints allow to obtain model-independent
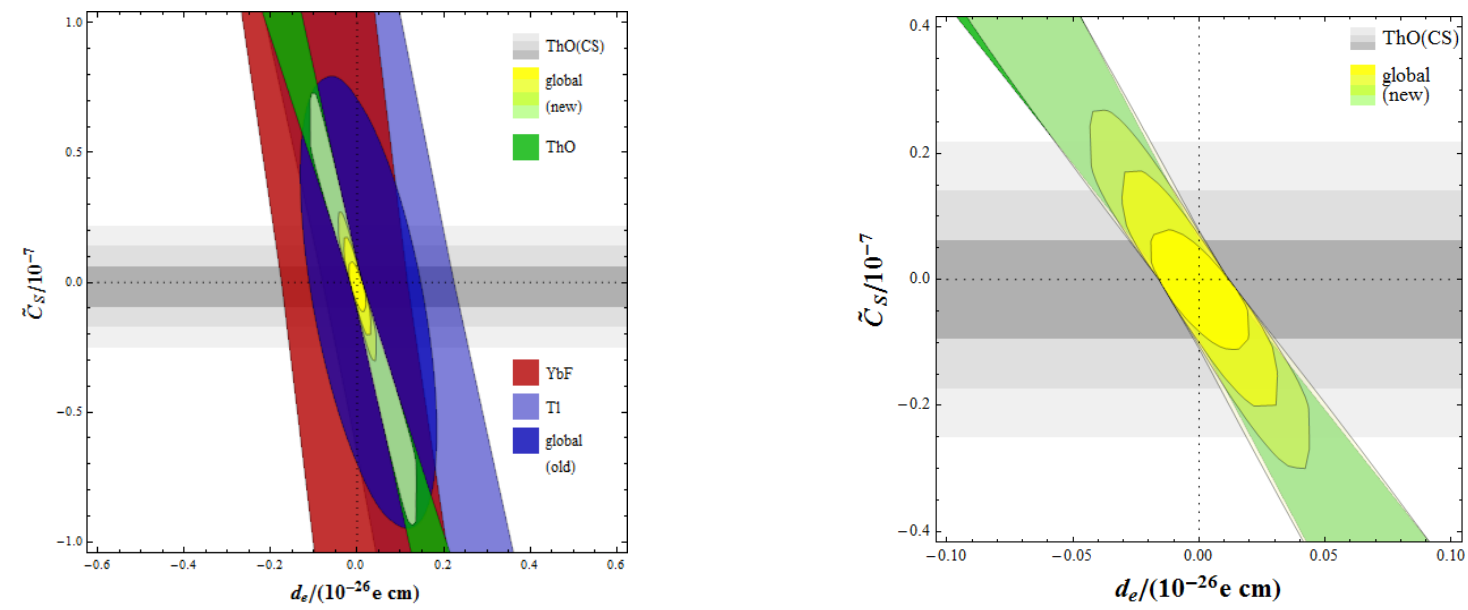

Figure 1: Fit to the recent measurements for paramagnetic systems [20-22], using additionally the $\mathrm{Hg}$ measurement [23] (blue ellipse on the left) or the assumption of limited cancellations in ThO with $n=1,2,3$ (grey bands, both plots), see text. Plots taken from Ref. [16].

constraints on the EDMs of all other heavy paramagnetic systems [18]; examples are

$d_{R b} \leq 3(0.5) \times 10^{-26} e \mathrm{~cm}, \quad d_{C s} \leq 10(2) \times 10^{-26} e \mathrm{~cm}, \quad d_{F r} \leq 60(20) \times 10^{-26} e \mathrm{~cm}($ all 95\% CL $)$.

A significant measurement in one of these systems larger than the first bound would indicate an experimental problem, while a value larger than the bound in brackets would indicate large cancellations between the two contributions. These limits are orders of magnitude below existing experimental ones [32-34]. Importantly, present experiments aim at an even better sensitivity [35-39].

The extension of this type of fit to all EDM measurements is clearly possible and has been proposed in Ref. [19]. While this is complicated by the many potential contributions - all of the terms in Eq. (2.1) are relevant in general -, it should be aimed at in the future. Since model-independent bounds/determinations are necessary to determine the specific structure of CP-violating NP contributions - and thereby potentially the model itself -, many measurements in different systems are very important. An additional complication for the EDMs of diamagnetic systems and neutrons is that the theoretical uncertainties for the relevant matrix elements are often large and can in some cases preclude the extraction of conservative limits, for instance on the cEDMs in Eq. (2.1) from $\mathrm{Hg}$ [16], further motivating complementary measurements.

\section{NP contributions to EDMs}

Reliable limits on parameters in NP models are difficult to achieve. Reasons are, apart from the fact discussed previously that presently less competetive measurements than relevant effective operators exist, the presence of several contributions to each of these coefficients and the various relevant hierarchies, i.e. mass scales, small mixing parameters and loop factors. This complicates 
semi-model-independent analyses for classes of models and allows strict statements only under additional assumptions.

Generic NP contributions at tree- and one-loop level are in conflict with the stringent experimental limits. On the two-loop level, usually so-called Barr-Zee- and Weinberg diagrams dominate [40-43], which compensate the additional loop factor by avoiding small mass factors. However, flavour sectors are usually far from "generic"; therefore in some cases also tree-level diagrams can be relevant, for example when they involve small mass factors, see below.

In order to demonstrate these qualitative statements in a specific model, we consider a general 2HDM, whose charged current Yukawa couplings can be parametrized in the Higgs basis as follows:

$$
\mathscr{L}_{Y}^{H^{ \pm}}=-\frac{\sqrt{2}}{v} H^{+}\left\{\bar{u}\left[V \varsigma_{d} M_{d} \mathscr{P}_{R}-\varsigma_{u} M_{u}^{\dagger} V \mathscr{P}_{L}\right] d+\bar{v} \varsigma_{l} M_{l} \mathscr{P}_{R} l\right\}+\text { h.c. },
$$

where the $M_{i}$ are diagonal mass matrices, $V$ denotes the CKM matrix, and the $\varsigma_{f}$ in principle arbitrary complex matrices. Below the constraints are given for the elements of these matrices, which can be translated into the parameters of any given 2HDM. To be specific and able to relate the resulting bounds also to those from other observables, we will furthermore consider the Aligned 2HDM (A2HDM) $[44,45]$, where the $\varsigma_{i}$ are complex numbers, thereby avoiding FCNCs on tree level while still allowing for a rich phenomenology including additional CP-violating phases. The couplings of the neutral Higgs mass-eigenstates $\varphi_{i}^{0}=\{h, H, A\}$ to a fermion species $f$ are denoted by $y_{f}^{\varphi_{i}}$; they depend not only again on the $\varsigma_{i}$, but in general additionally on the parameters of the Higgs potential, severely complicating the analysis. These couplings fulfill the relations

$$
\sum_{i} \operatorname{Re}\left(y_{f}^{\varphi_{i}^{0}}\right) \operatorname{Im}\left(y_{f^{\prime}}^{\varphi_{0}^{0}}\right)= \pm \operatorname{Im}\left[\left(\varsigma_{F(f)}^{*}\right)_{f f}\left(\varsigma_{F\left(f^{\prime}\right)}\right)_{f^{\prime} f^{\prime}}\right],
$$

with a vanishing right-hand side for real $\varsigma_{i}$ (as e.g. the case for $\mathscr{Z}_{2} 2 \mathrm{HDM}$ models) or $f=f^{\prime}$ (more generally fermions of the same family if the $\varsigma_{i}$ are family-universal as $e . g$. in the A2HDM). Importantly, the right-hand side is independent of the parameters of the scalar potential. While in practical calculations there are mass-dependent weight factors in the sum on the left, the relation still holds exactly in two limits: trivially when the neutral scalars are degenerate, but also in the decoupling limit [16]. Therefore, in general cancellations can be expected for any mass spectrum and the influence of $\mathrm{CP}$ violation in the potential is reduced. Clearly, this observation provides a protection against large EDMs for models which exhibit new CP-violating parameters in the potential, only. Below we will assume this cancellation to occur and evaluate the right-hand side with a common weight factor at an intermediate effective neutral Higgs mass $\bar{M}_{\varphi}$.

In this setup, the situation is typically the one described above: four-quark (tree-level) contributions are subleading, one-loop contributions to (colour-)EDMs are under control (but not necessarily tiny), and two-loop contributions are dominant, but also the tree-level quark-electron couplings are relevant, despite the small mass factors $[16,46]$.

The electron EDM receives contributions mostly from Barr-Zee diagrams. The resulting constraints on $\operatorname{Im}\left(\varsigma_{u, 33} \varsigma_{l, 11}^{*}\right)$ demonstrate the strength of this observable: $\operatorname{Im}\left(\varsigma_{u, 33}^{*} \varsigma_{l, 11}\right) \lesssim 0.05$ (for $n=1)$. This already questions the common assumptions that these factors are $\mathscr{O}(1)$. In the A2HDM this can be compared to the absolute value of this parameter combination obtained from leptonic and semileptonic decays [45,47], which is about a factor 1000 weaker. 
As mentioned above, also the constraint from $\tilde{C}_{S}$ is relevant: while in this case the constraint seems much weaker, $\operatorname{Im}\left(\varsigma_{d, 11}^{*} \varsigma_{l, 11}\right) \lesssim 15$, the bound on the ratio with $\bar{M}_{\varphi}^{2}$ is again at least a factor 100 stronger than the corresponding one with the charged-Higgs mass from (semi-)leptonic processes [45, 47].

For the neutron, the constraint induced in the charged-Higgs sector via the Weinberg operator is the dominant one, leading to $\operatorname{Im}\left(\varsigma_{u, 33}^{*} \varsigma_{l, 33}\right) \lesssim 1$. While this does not imply any sizable finetuning, it already prohibits large CP-violating effects in other observables in specific models. For instance, while the indirect constraint from the branching ratio in $b \rightarrow s \gamma$ in the A2HDM still allows for a sizable CP asymmetry for this process, a NP contribution of $\left|A_{\mathrm{CP}}(b \rightarrow s \gamma)\right| \lesssim 1 \%$ follows from the EDM bound and the discussion in Refs. [48,49].

These examples show the potential of EDMs, but also their complementarity to other searches, since only the imaginary parts of parameter combinations are constrained. However, for the combinations EDMs are sensitive to, they are often the strongen constraints available.

\section{Conclusions}

EDMs provide unique constraints for the CP-violating sectors of NP models. A potential discovery of a non-vanishing fundamental EDM in any system would be a major achievement, independent of its source. The interpretation of bounds and potential measurements in terms of fundamental theory parameters requires the careful estimation of theoretical uncertainties and is complicated by potential cancellations on various levels. While this problem can be addressed for heavy paramagnetic systems to extract the electron EDM and scalar electron-nucleon coupling model-independently, a similar approach including all relevant systems should be aimed at, but requires several additional measurements for different systems.

For the occuring combinations of parameters, EDMs typically provide the most stringent constraints. We demonstrated this explicitly for general 2HDMs, and more specifically for the A2HDM, where large CP-violating effects in other observables are strongly bounded by the existing EDM limits. Given the present strength of the constraints, forthcoming experiments will test a crucial part of the parameter space and might turn existing bounds into observations.

\section{References}

[1] M. Kobayashi and T. Maskawa. CP violation in the renormalizable theory of weak interaction. Prog. Theor. Phys., 49:652, 1973.

[2] S. L. Glashow, J. Iliopoulos, and L. Maiani. Weak Interactions with Lepton-Hadron Symmetry. Phys. Rev., D2:1285-1292, 1970.

[3] R.D. Peccei and Helen R. Quinn. CP Conservation in the Presence of Instantons. Phys.Rev.Lett., 38:1440-1443, 1977.

[4] Steven Weinberg. A New Light Boson? Phys. Rev. Lett., 40:223-226, 1978.

[5] Frank Wilczek. Problem of Strong P and T Invariance in the Presence of Instantons. Phys. Rev. Lett., 40:279-282, 1978.

[6] I.B. Khriplovich and A.R. Zhitnitsky. What Is the Value of the Neutron Electric Dipole Moment in the Kobayashi-Maskawa Model? Phys.Lett., B109:490, 1982. 
[7] M.B. Gavela, A. Le Yaouanc, L. Oliver, O. Pene, J.C. Raynal, et al. CP Violation Induced by Penguin Diagrams and the Neutron Electric Dipole Moment. Phys.Lett., B109:215, 1982.

[8] Bruce H.J. McKellar, S.R. Choudhury, Xiao-Gang He, and Sandip Pakvasa. The Neutron Electric Dipole Moment in the Standard KM Model. Phys.Lett., B197:556, 1987.

[9] Thomas Mannel and Nikolai Uraltsev. Loop-Less Electric Dipole Moment of the Nucleon in the Standard Model. Phys.Rev., D85:096002, 2012.

[10] M. E. Pospelov and I. B. Khriplovich. Electric dipole moment of the W boson and the electron in the Kobayashi-Maskawa model. Sov. J. Nucl. Phys., 53:638-640, 1991. [Yad. Fiz.53,1030(1991)].

[11] Michael J. Booth. The Electric dipole moment of the W and electron in the Standard Model. 1993. hep-ph/9301293.

[12] Maxim Pospelov and Adam Ritz. CKM benchmarks for electron EDM experiments. Phys.Rev., D89:056006, 2014.

[13] A.D. Sakharov. Violation of CP Invariance, c Asymmetry, and Baryon Asymmetry of the Universe. Pisma Zh.Eksp.Teor.Fiz., 5:32-35, 1967.

[14] Maxim Pospelov and Adam Ritz. Electric dipole moments as probes of new physics. Annals Phys., 318:119-169, 2005.

[15] Jonathan Engel, Michael J. Ramsey-Musolf, and U. van Kolck. Electric Dipole Moments of Nucleons, Nuclei, and Atoms: The Standard Model and Beyond. Prog.Part.Nucl.Phys., 71:21-74, 2013.

[16] Martin Jung and Antonio Pich. Electric Dipole Moments in Two-Higgs-Doublet Models. JHEP, 1404:076, 2014.

[17] V. A. Dzuba, V. V. Flambaum, and C. Harabati. Relations between matrix elements of different weak interactions and interpretation of the parity-nonconserving and electron electric-dipole-moment measurements in atoms and molecules. Phys. Rev. A, 84:052108, 2011. Erratum ibid, 85, 029901 (2012).

[18] Martin Jung. A robust limit for the electric dipole moment of the electron. JHEP, 1305:168, 2013.

[19] Timothy Chupp and Michael Ramsey-Musolf. Electric Dipole Moments: A Global Analysis. Phys. Rev., C91(3):035502, 2015.

[20] Jacob Baron et al. Order of Magnitude Smaller Limit on the Electric Dipole Moment of the Electron. Science Magazine, 343 (6168):269-272, 2014.

[21] J.J. Hudson, D.M. Kara, I.J. Smallman, B.E. Sauer, M.R. Tarbutt, et al. Improved measurement of the shape of the electron. Nature, 473:493-496, 2011.

[22] B. C. Regan, E. D. Commins, C. J. Schmidt, and D. DeMille. New limit on the electron electric dipole moment. Phys. Rev. Lett., 88:071805, 2002.

[23] W. C. Griffith et al. Improved Limit on the Permanent Electric Dipole Moment of Hg-199. Phys. Rev. Lett., 102:101601, 2009.

[24] C. A. Baker et al. An improved experimental limit on the electric dipole moment of the neutron. Phys. Rev. Lett., 97:131801, 2006.

[25] S. Afach et al. A Revised Experimental Upper Limit on the Electric Dipole Moment of the Neutron. 2015. 
[26] A.P. Serebrov, E.A. Kolomenskiy, A.N. Pirozhkov, I.A. Krasnoschekova, A.V. Vassiljev, et al. New measurements of the neutron electric dipole moment. JETP Lett., 99:4-8, 2014.

[27] I.B. Khriplovich and S.K. Lamoreaux. CP violation without strangeness: Electric dipole moments of particles, atoms, and molecules. Springer-Verlag Berlin Heidelberg New York, 1997.

[28] L. I. Schiff. Measurability of Nuclear Electric Dipole Moments. Phys. Rev., 132:2194-2200, 1963.

[29] P. G. H. Sandars. The electric dipole moment of an atom. Physics Letters, 14(3):194, 1965.

[30] P. G. H. Sandars. Enhancement factor for the electric dipole moment of the valence electron in an alkali atom. Physics Letters, 22(3):290-291, 1966.

[31] V.V Flambaum. On Enhancement of the electron Electric Dipole Moment in Heavy Atoms. Yad.Fiz., 24:383-386, 1976.

[32] E. S. Ensberg. Experimental upper limit for the permanent electric dipole moment of $\mathrm{rb}^{85}$ by optical-pumping techniques. Phys. Rev., 153:36-43, 1967.

[33] F.R. Jr. Huang-Hellinger. A Search for a Permanent Electric Dipole Moment in Rubidium. PhD thesis, University of Washington, Seattle, 1987. Quoted in [27], unpublished.

[34] S. A. Murthy, D. Krause, Z. L. Li, and L. R. Hunter. New Limits on the Electron Electric Dipole Moment from Cesium. Phys. Rev. Lett., 63:965-968, 1989.

[35] D.S. Weiss, F. Fang, and J. Chen. Measuring the electric dipole moment of Cs and Rb in an optical lattice. Bull.Am.Phys.Soc., APR03:J1.008, 2003. For the present status, see the talk by D. Weiss at the "EDM searches at Storage Rings" workshop at ECT*, http: / / www . ect star. eu.

[36] Jason M. Amini, Charles T. Munger, Jr., and Harvey Gould. Electron electric dipole moment experiment using electric- field quantized slow cesium atoms. Int. J. Mod. Phys., D16:2337-2342, 2008.

[37] M. Kittle, T. Burton, L. Feeney, and D. J. Heinzen. New experiment to measure the electron electric dipole moment. APS Division of Atomic, Molecular and Optical Physics Meeting Abstracts, pages $1056 \mathrm{P}-+, 2004$.

[38] Y. Sakemi, K. Harada, T. Hayamizu, M. Itoh, H. Kawamura, et al. Search for a permanent EDM using laser cooled radioactive atom. J.Phys.Conf.Ser., 302:012051, 2011.

[39] B. J. Wundt, C. T. Munger, and U. D. Jentschura. Quantum dynamics in atomic-fountain experiments for measuring the electric dipole moment of the electron with improved sensitivity. Phys. Rev. X, 2:041009, 2012. See also http://eedm. info/index.html.

[40] Steven Weinberg. Larger Higgs Exchange Terms in the Neutron Electric Dipole Moment. Phys. Rev. Lett., 63:2333, 1989.

[41] Duane A. Dicus. Neutron electric dipole moment from charged higgs exchange. Phys. Rev., D41:999, 1990.

[42] Stephen M. Barr and A. Zee. Electric dipole moment of the electron and of the neutron. Phys. Rev. Lett., 65:21-24, 1990. Erratum-ibid.65:2920,1990.

[43] J. F. Gunion and D. Wyler. Inducing a large neutron electric dipole moment via a quark chromoelectric dipole moment. Phys. Lett., B248:170-176, 1990.

[44] Antonio Pich and Paula Tuzon. Yukawa Alignment in the Two-Higgs-Doublet Model. Phys. Rev., D80:091702, 2009. 
[45] Martin Jung, Antonio Pich, and Paula Tuzon. Charged-Higgs phenomenology in the Aligned two-Higgs- doublet model. JHEP, 11:003, 2010.

[46] Andrzej J. Buras, Gino Isidori, and Paride Paradisi. EDMs vs. CPV in $B_{s, d}$ mixing in two Higgs doublet models with MFV. Phys. Lett., B694:402-409, 2011.

[47] Alejandro Celis, Martin Jung, Xin-Qiang Li, and Antonio Pich. Sensitivity to charged scalars in $B \rightarrow D^{(*)} \tau \nu_{\tau}$ and $B \rightarrow \tau \nu_{\tau}$ decays. JHEP, 1301:054, 2013.

[48] Martin Jung, Antonio Pich, and Paula Tuzon. The B $\rightarrow$ Xs gamma Rate and CP Asymmetry within the Aligned Two-Higgs-Doublet Model. Phys. Rev., D83:074011, 2011.

[49] Martin Jung, Xin-Qiang Li, and Antonio Pich. Exclusive radiative B-meson decays within the aligned two-Higgs-doublet model. JHEP, 1210:063, 2012. 